ENFOQUES JURÍDICOS

REVISTA MULTIDISCIPLINAR DEL CEDECS

ISSN 26832070

Número 04

julio-diciembre 2021
Artículo: "La reforma en materia de teletrabajo en México"

Jorge Martínez Martínez

DOI: https://doi.org/10.25009/ej.v0i04.2571

\title{
La reforma en materia de teletrabajo en México
}

Recibido 19 marzo 2021-Aceptado 01 junio 2021

\author{
Jorge Martínez Martínez \\ Universidad Veracruzana. Xalapa-Veracruz, México \\ jormartinez@uv.mx
}

RESUMEN: La transformación de las relaciones de trabajo derivada de la flexibilidad laboral ha sido una realidad desde hace ya varias décadas; por ello las normas de trabajo deben ser dinámicas y adaptarse a las circunstancias que se van presentando, como respuesta no sólo a una tendencia, sino a la permanente búsqueda del equilibrio entre los factores de la producción como una de las finalidades fundamentales del derecho del trabajo.

La pandemia de COVID-19 ha servido de catalizador para hacer realidad la normativa en materia de teletrabajo en México, así como ha sucedido en algunos otros países del mundo, ya que la regulación era insuficiente, y dejaba al teletrabajo subsumido dentro del trabajo a domicilio.

En el presente, se hace un análisis de la reforma llevada a cabo en fecha 11 de enero del año 2021, de su regulación, parte de su
ABSTRACT: The transformation of labor relations derived from labor flexibility has been a reality for several decades now; therefor, labor rules must be dynamic and adaptive to the circumstances that arise, in response not only to a trend, but also to the permanent search for balance between the factors of production as one of the fundamental purposes of the labor law.

COVID-19 pandemic has served as catalyzer to teleworking rules on Mexico became a reality, as happened in other countries over the world, due to the current regulations were insufficient, which subsumed teleworking on within working at domicile rules.

This work is an analysis of the January $11^{\text {th }}$ 2021 reform, its regulation, part of its problematic and challenges or opportunity areas which needs to be outdone to reach the accomplishment of special obligations of

\footnotetext{
* Doctor en Derecho Público por el Instituto de Investigaciones Jurídicas de la Universidad Veracruzana, Investigador Nacional Nivel I del Sistema Nacional de Investigadores del CONACyT, catedrático de la Facultad de Derecho de la Universidad Veracruzana.
}

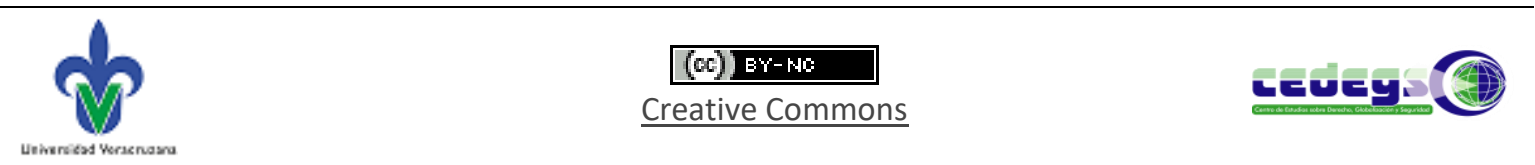


ENFOQUES JURIDICOS

REVISTA MULTIDISCIPLINAR DEL CEDECS

ISSN 26832070

Número 04

julio-diciembre 2021
Artículo: "La reforma en materia de teletrabajo en México"

Jorge Martínez Martínez

DOI: https://doi.org/10.25009/ej.v0i04.2571 problemática y los retos o áreas de oportunidad que deben superarse para lograr el cumplimiento de las obligaciones especiales que implica la reforma; pues ésta debe responder ante una realidad compleja que indudablemente ha transformado las relaciones de trabajo y los procesos productivos.

Palabras clave: Teletrabajo, flexibilización laboral, Industria 4.0, pandemia de COVID-19. the reform; as it shall response to a complex reality which undoubtedly has transform the working relations and production processes. Keywords: Teleworking, labor flexibility, industry 4.0, COVID-19 pandemic.

SUMARIO: Introducción. 1. La transformación de las relaciones de trabajo; 2. La flexibilización laboral. 3. El trabajo a domicilio. 4. El teletrabajo en el contexto de la pandemia de COVID-19. 5. La reforma a la LFT en materia de teletrabajo. Conclusiones. Fuentes de consulta.

\section{Introducción}

Las relaciones de trabajo han cambiado a través del tiempo, se adaptan a un contexto globalizado, con ello, han ido surgiendo la flexibilización laboral, así como las nuevas formas de contratación, tales como el teletrabajo o los contratos a prueba y de capacitacion inicial (entre otras) que han motivado incluso reformas como la del año 2012 que reguló, estas cuestiones que ya se suscitaban en la práctica; no obstante, en muchos de los casos se tradujeron en violaciones a los derechos de los trabajadores.

En fecha 11 de enero del año 2021 se publicó en el Diario Oficial de la Federación el Decreto por el cual se reforma el artículo 311 y se adiciona el capítulo XII bis de la Ley Federal del Trabajo en materia de teletrabajo.

Reforma que se considera pertinente, máxime en estos tiempos en donde la pandemia ocasionada por el COVID-19, ha transformado las relaciones de trabajo, en una adaptación a las circunstancias que se presentan.

No obstante lo anterior, surgen retos para poder materializar una reforma como la mencionada, se aprecian dificultades para el cumplimiento de las obligaciones derivadas de esta modalidad de trabajo; que si bien había quedado pendiente, ya se consideraba necesaria por lo menos desde la anterior reforma a la ley de la materia.

\section{La transformación de las relaciones de trabajo}

La evolución de la sociedad, la interacción o dependencia tecnológica, la economía y la búsqueda de competitividad, han condicionado o han sido algunos factores, entre otros, para que las relaciones de trabajo se transformen, lo que ha originado que la realidad se

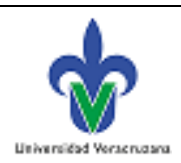

pp. $61-83$ 
ENFOQUES JURIDICOS

REVISTA MULTIDISCIPLINAR DEL CEDECS

ISSN 26832070

Número 04

julio-diciembre 2021
Artículo: "La reforma en materia de teletrabajo en México"

Jorge Martínez Martínez

DOI: https://doi.org/10.25009/ej.v0i04.2571

imponga y con ello hayan surgido desde hace algún tiempo la flexibilización del trabajo y las nuevas formas de contratación como son el contrato a prueba, de capacitación inicial y el trabajo de temporada; cambios en las relaciones de trabajo que han conducido a la flexibilización de los derechos laborales, ya que además no puede haber conquistas sindicales cada vez mayores, al punto que lleguen a ser perjudiciales para la fuente de trabajo.

La pandemia de COVID-19 ha impuesto circunstancias a las relaciones de trabajo, y ha traído consigo algunas repercusiones, que se aprecian no sólo en la cotidianidad, sino también se han expresado en la Guía Práctica El teletrabajo durante la pandemia de COVID19 y después de ella, publicada por la Organización Internacional del Trabajo.

Aunque la cantidad de personas que teletrabajan a tiempo parcial o a tiempo completo ha ido aumentando gradualmente a lo largo de los años (Eurostat, 2018), sin duda la pandemia ha acelerado la adopción, por parte de los empleadores, de las modalidades de teletrabajo. En un contexto como el de la pandemia de COVID-19, el teletrabajo ha demostrado ser una herramienta importante para garantizar la continuidad operativa. Mientras, en circunstancias normales, sus beneficios incluyen la reducción del tiempo de desplazamiento, la posibilidad de que los trabajadores se centren en sus tareas lejos de las distracciones de la oficina y una oportunidad para lograr un mejor equilibrio entre la vida laboral y profesional. El teletrabajo permite a los trabajadores tener un horario más flexible y la libertad de trabajar fuera de las instalaciones del empleador. También puede conllevar riesgos que deben preverse y prevenirse, como el aislamiento (en particular para las personas que viven solas) y la pérdida de contacto con los compañeros de trabajo. (Organización Internacional del Trabajo [OIT] 2021)

En tal sentido, el teletrabajo ha contribuido a la realización o materialización del trabajo decente, en los casos en que permite un equilibrio del trabajador en todo el cúmulo de actividades que integran su vida; aunque en algunos otros ha sido un reto no superado y ha ocasionado consecuencias negativas.

El teletrabajo es un concepto relativamente reciente que llegó para quedarse, pues el día de hoy es una modalidad de trabajo necesaria; particularmente con la pandemia de COVID-19, no sólo ha cobrado especial relevancia, sino en el caso mexicano se ha incorporado al derecho vigente a partir del 11 de enero del año 2021; a pesar de que en la práctica ya era observable, ello ha traído consecuencias y algunas complicaciones, como es el caso de la determinación de los riesgos de trabajo que pueden suscitarse, y aunque parecieran ejemplos de laboratorio; en el caso, es posible establecer la relación que guarda el medio ambiente de trabajo, con el accidente o enfermedad profesional.

Igualmente algunas de las dificultades que pueden mencionarse, sin ánimo de exahustividad es la determinación acerca de si el trabajo realizado pertenece a la categoría de teletrabajo materialmente, es decir si en la práctica se subsume el tipo de relación que se trate con la descripción legal; ya que no necesariamente el uso de las tecnologías de información y comunicación otorga per se la naturaleza jurídica de teletrabajo.

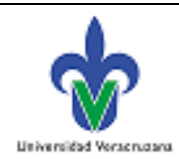

pp. $61-83$ 
ENFOQUES JURIDICOS

REVISTA MULTIDISCIPLINAR DEL CEDEGS

ISSN 26832070

Número 04

julio-diciembre 2021
Artículo: "La reforma en materia de teletrabajo en México"

Jorge Martínez Martínez

DOI: https://doi.org/10.25009/ej.v0i04.2571

Más adelante surgirán también para los patrones, algunos inconvenientes, como lo son los costos que para la empresa implica la reforma que se analiza, y el proporcionar los elementos que requiere el teletrabajador y que cumplan con las especificaciones necesarias. Sobre todo en el presente contexto derivado de la pandemia de COVID 19, con un aumento considerable en el precio de los insumos tecnológicos.

Para comenzar el estudio, hay que referirse al concepto de teletrabajo, que surge en Estados Unidos en el año de 1973, tal como lo señala Julio Ismael Camacho:

El concepto de teletrabajo nace en Estados Unidos, en 1973, partir de un grupo de científicos de la University of Southern California dirigidos por el físico Jack Niles. Dicho grupo, en el marco de la crisis del petróleo, investigó la aplicación del trabajo de las (entonces disponibles) tecnologías informáticas para reducir el traslado de trabajadores a las oficinas, reemplazándolo por el traslado de la información necesaria para que el trabajador realice sus tareas desde su casa o centro de teletrabajo próximo su domicilio (Camacho, 2021: 128).

El autor de referencia agrega que ello traería ventajas como las reducciones al tránsito vehicular y por ende la disminución en la contaminación derivada de la emisión de gases tóxicos al ambiente, por lo que desde aquel momento ya se visualizaban enormes ventajas para el teletrabajo, que hoy en día siguen observándose.

En ocasiones se ha pensado que existen actividades en donde lo más propicio no es el teletrabajo, pero que ante contextos como el actual, se ha presentado una adaptación o conversión forzada hacia tal modalidad; a modo de ejemplo y aunque en sentido estricto no se trata de teletrabajo, se evidencia que para los trabajadores de la educación no existió la capacitación deseable o por lo menos necesaria para llevar a cabo las actividades de manera virtual.

El trabajo y el comercio guardan estrecha relación, debido a que son las empresas y la actividad económica, las que requieren, al igual que los servicios, de la mano de obra que proporcionan los trabajadores, por lo que el contexto de la pandemia ha modificado también el comercio, y ha significado oportunidades para los tele trabajadores:

En América Latina en los tres primeros meses de la pandemia tuvimos 13 millones adicionales como primeros usuarios en el ecommerce, esto significa que 2 de cada 10 usuarios son nuevos o están usándolo por primera vez, indicó Luz Adriana Ramírez, directora general de Visa México, en conferencia de prensa. (Lozano, 2020)

Esta modalidad de trabajo requiere de las competencias adecuadas que les permitan a los trabajadores la realización de sus actividades, por lo cual habría ventaja para los jóvenes, o los denominados nativos digitales, ya adaptados a las tecnologías de la información y la comunicación, pues la brecha generacional puede constituir un obstáculo para los de mayor edad.

Es imprescindible que ante estas circunstancias, deban de adaptarse las normas de trabajo para regular el teletrabajo en el marco de la ley de la materia; no obstante, al igual que sucede con cualquier reforma, la normativa per se no puede producir los cambios de

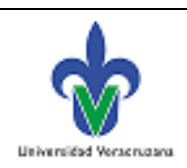

pp. $61-83$

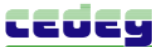


ENFOQUES JURIDICOS

REVISTA MULTIDISCIPLINAR DEL CEDECS

ISSN 26832070

Número 04

julio-diciembre 2021
Artículo: "La reforma en materia de teletrabajo en México"

Jorge Martínez Martínez

DOI: https://doi.org/10.25009/ej.v0i04.2571

manera instantánea, sino que el cumplimiento de estas disposiciones supondrá una área de oportunidad, que debe irse superando en la medida en que la voluntad y las posibilidades tanto de los agentes económicos, como de los trabajadores lo permitan.

A través del tiempo, han surgido transformaciones en los modelos de producción, como resultado de la evolución de la sociedad y de la incorporación de mecanismos, instrumentos, métodos y modelos que han significado cambios fundamentales en la industria, comercio o servicios.

Para ello hay que referirse a dos aspectos torales, que son los mencionados modelos y las revoluciones industriales, aquellos son entendidos como las formas en las que se organiza la actividad económica en un contexto determinado, que repercute necesariamente en sus resultados.

A través del tiempo, han surgido dos grandes modelos de producción que han transformado profundamente las relaciones de trabajo, ambos han emergido desde la industria automotriz y permeado a todas las demás actividades; son el fordismo y el toyotismo, el primero se caracteriza por la división de la producción en etapas, para lograr grandes volúmenes en el menor tiempo posible, como consecuencia de la división y especialización del trabajo, este modelo se instauró a partir de la Segunda Guerra Mundial y se mantuvo vigente hasta los años setentas (Piñero, 2004: 2).

El segundo de los modelos de producción es el toyotismo, que reemplazó al fordismo y se caracteriza por el trabajo en grupos y en producir "just on time", por lo que la producción masiva se sustituye por la oferta diversificada de acuerdo a los gustos y necesidades de cada cliente, además de que se busca perfeccionar la producción, la polivalencia de los trabajadores; dicho modelo se instaura desde los años setenta, se consolida en los ochentas y se mantiene vigente hasta nuestros días. (Zuccarino, 2012: 202)

Por otro lado los cambios en los modelos de producción, así como la incorporación de las herramientas y la tecnología, han permitido la clasificación de las revoluciones industriales, en cuatro, la primera caracterizada por la mecanización de los procesos productivos, a partir de la invención por parte de James Watt de la máquina de vapor en el año de 1788, en donde el carbón se convierte en materia prima en sustitución de la madera como fuente de energía, aunque también puede señalarse que la Industria 1.0, surge desde el año de 1700 en Inglaterra a partir de los cambios observados en la industria textil y el transporte del agua. (Taskan, Karatov \& Kubat, 2020: 81)

La segunda revolución industrial, denominada también Industria 2.0; se caracterizó por la electrificación de los procesos productivos, y surgió como resultado de los cambios en las fuentes de energía, se utiliza petróleo, acero, electricidad, minerales, máquinas de combustión interna, el teléfono, la radio, la iluminación en general, la máquina de escribir, la prensa escrita, así como el abaratamiento en la producción del acero a partir del año de 1860, por parte del inventor británico H. Bessemer. (Taskan, Karatov \& Kubat, 2020: 81)

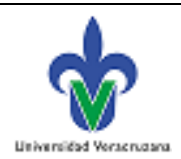

pp. $61-83$ 
ENFOQUES JURIDICOS

REVISTA MULTIDISCIPLINAR DEL CEDECS

ISSN 26832070

Número 04

julio-diciembre 2021
Artículo: "La reforma en materia de teletrabajo en México"

Jorge Martínez Martínez

DOI: https://doi.org/10.25009/ej.v0i04.2571

La Industria 3.0 o tercera revolución industrial, situada aproximadamente en el año de 1970, se caracterizó por la automatización y digitalización de la producción, lo cual fue el resultado del descubrimiento y aplicación de la energía nuclear y la invención de la computadora, con lo cual hoy en día se realizan la mayor parte de los procesos de producción de la industria y sin los cuales no podrían siquiera entenderse. En este período surgen grandes innovaciones como el láser, la biogenética, energía nuclear, materiales sintéticos, fibra óptica, computadoras, tecnología y comunicaciones, (Taskan, Karatov \& Kubat, 2020: 81) aunque no todo han sido avances, pues la gran dependencia de los combustibles fósiles ha impactado en el cambio climático y comprometido el desarrollo sustentable.

La última revolución es la denominada Industria 4.0, que surge después del último cuarto del siglo XX, o bien se señala el año 2000; en donde se aprecian cambios tecnológicos de manera frecuente, con avances como la internet, las redes inalámbricas, software, automatización, mecatrónica y robótica; (Tepe \& Mucan, 2021: 160) todo lo que se incorpora cada vez más a la vida cotidiana, actualmente se puede apreciar cada vez más la automatización de los procesos productivos, así como también la presencia de la tecnología en casi todos los ámbitos del trabajo y de la vida privada; incluso la OIT señaló algunos cambios que con motivo de la pandemia de COVID-19 se suscitaron en Japón:

Cuando el coronavirus comenzó a propagarse en Japón, el Gobierno declaró el estado de emergencia en las principales ciudades e instó a la población a reducir el contacto entre las personas por lo menos en un $70 \%$. Por consiguiente, se exigió a los empleadores que pasaran al trabajo en línea. Uno de los principales grupos de telecomunicaciones es partidario del teletrabajo hace varios años $y$, desde febrero de 2020, ha instado a los empleados a evitar los desplazamientos en las horas pico o que simplemente trabajen desde casa, para proteger a sus 200000 empleados en todo Japón del creciente brote de coronavirus en el país. La empresa también comenzó a utilizar robots avatar en abril de 2016 como parte de sus esfuerzos para promover el teletrabajo, una política destinada a permitir que los empleados sigan trabajando mientras crían a sus hijos o cuidan de sus padres ancianos. Usando los robots avatares, los trabajadores remotos pueden ver su oficina y comunicarse con sus colegas. Los robots miden aproximadamente $20 \mathrm{~cm}$ de altura con cámara, micrófono y altavoces incorporados, junto con una aplicación de "teletrabajo" instalada en un teléfono o un iPad, son operados a distancia por el teletrabajador y pueden ser llevados por el personal de la oficina. Incluso, pueden asistir a reuniones en nombre de los empleados remotos. Además, es posible manejar las manos y la cabeza del robot, incluso utilizando la comunicación no verbal con diversos gestos, desde aplausos hasta saludos. "Puedo sentir el ambiente en la oficina y es como si estuviera allí", dijo un teletrabajador de unos 40 años. (OIT, 2021)

El impacto de la Industria 4.0 aunado a los modelos de producción, ha sido fundamental para las empresas y para la vida personal, ya que gracias la tecnología con la que cuenta cada país, es que se han superado algunas de las consecuencias derivadas de la pandemia de COVID-19, pues esto es lo que posibilitó teletrabajar; del mismo modo saltan a la vista las enormes diferencias que existen con los Estados menos tecnificados o con menor capacidad económica para transformar sus actividades.

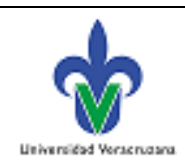

pp. $61-83$ 
ENFOQUES JURIDICOS

REVISTA MULTIDISCIPLINAR DEL CEDECS

ISSN 26832070

Número 04

julio-diciembre 2021
Artículo: "La reforma en materia de teletrabajo en México"

Jorge Martínez Martínez

DOI: https://doi.org/10.25009/ej.v0i04.2571

Una vez que se han puntualizado los cambios que han producido los modelos de producción y las revoluciones industriales, en conjunto se evidencia que la dependencia tecnológica ha llegado para quedarse en las relaciones de trabajo, pues hasta en las labores que llegan a ser completamente manuales, se incorpora la tecnología, por ejemplo para el registro de asistencia, para el pago de salarios, para las cuestiones de seguridad social como lo son las altas y bajas de los trabajadores, etc.

\section{La flexibilización laboral}

La flexibilización laboral se entiende como la maleabilidad que ha permeado las relaciones de trabajo para adaptarse a las circunstancias complejas de acuerdo al entorno, que permitan superar las dificultades en las empresas o establecimientos.

De acuerdo a Freddy Arancibia Fernández, (2011) existen cuatro grandes dimensiones que compondrían el concepto de flexibilidad laboral, que son: la primera, flexibilidad de la organización productiva, denominada igualmente flexibilidad organizativa o de la contratación externa y/o externalización, por lo que en esta queda comprendida la subcontratación; la segunda es la flexibilidad de la organización del trabajo, para que la empresa se adapte a las variaciones del mercado, por lo que encuentra relación con la nueva ola de la gerencia:

De este modo la ruptura del one best way hace que el know how, usurpado a los trabajadores por la Organización Científica del Trabajo -desarrollada por Frederick W. Taylor, profundizada en base a la línea montaje por Henry Ford, y plasmada como modelo administrativo por Henry Fayol- vuelva a los primeros, propiciando el surgimiento de lo que algunos han denominado el "nuevo artesanado" (De la Garza, 2003) o "trabajador flexible" (Chávez, 2001)." (Arancibia, 2011: 43)

La organización de la producción, tiene que ver entonces con los modelos de producción que han respondido a las necesidades de cada época, se han impuesto como tendencia y han influido no sólo en el trabajo, sino en general en todo tipo de organizaciones, que permiten, en estricto sentido mejorar la eficiencia.

La tercera acepción de la flexibilidad es la de la gestión productiva, que se refiere según Arancibia: "[...] a la capacidad tecnológica de una empresa -principalmente a la relacionada con la microelectrónica- para alterar u corregir tanto su proceso de producción interno, como la cantidad y el tipo de productos finales." (Arancibia, 2011: 44) Lo que permite superar la gama de productos y los errores, además de la disminución de costos.

La última de las dimensiones de la flexibilidad es la del mercado laboral o mercado de trabajo:

La flexibilidad del mercado laboral o flexibilidad del mercado de trabajo (De la Garza, 2003; Chávez, 2001; Agacino, et. al., 1998) dice tener relación con aquellos aspectos de la flexibilidad laboral que se encuentran posibilitados por la legislación laboral de un país o región y cuyo objetivo es dotar a las empresas de elevados márgenes de movilidad en las relaciones laborales que genera con sus trabajadores. Dentro de este tipo de flexibilidad podemos encontrar las denominadas flexibilidad



pp. $61-83$

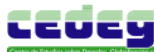


ENFOQUES JURIDICOS

REVISTA MULTIDISCIPLINAR DEL CEDECS

ISSN 26832070

Número 04

julio-diciembre 2021
Artículo: "La reforma en materia de teletrabajo en México"

Jorge Martínez Martínez

DOI: https://doi.org/10.25009/ej.v0i04.2571

interna y flexibilidad externa, ambas con sus propios componentes o sub-dimensiones. (Arancibia, 2011: 45)

De manera que la interna comprende a las condiciones de trabajo, y por ende a la salarial, la de la jornada u horarios de trabajo y a la funcional.

La flexibilidad salarial se refiere a la variablidad del salario en función de la productividad del trabajador, de manera que su salario puede integrarse únicamente con elementos variables o bien con elementos fijos y variables de manera combinada, siempre que se respeten los mínimos legales, lo cual conduce a contar con trabajadores que le ponen mayor empeño a su trabajo, pues su remuneración aumenta en la medida en que sean más productivos, o por el contrario se reduce.

Esto desde luego siempre ha existido, pues así acontece con el trabajo por unidad de obra o a destajo, el cual a través del tiempo ha resultado muy conveniente para los factores de la producción, aunque desde luego esto depende de la propia naturaleza del trabajo.

La especialización del trabajo en muchos de los casos requiere que los trabajadores cuenten con competencias específicas que los habiliten para la realización de ciertas actividades. Lo que puede limitar esta polivalencia; no obstante si no se requiere gran especialización esta multihabilidad reduce costos y vuelve benéfica a la flexibilidad funcional.

En lo que respecta a la flexibilidad externa, comprende la variación numérica que permite la contratación de personal dependiendo el aumento de las necesidades en la fuente de trabajo, que igualmente siempre ha existido, pues este es el caso de los trabajadores de temporada, como puede ser la relativa a las festividades navideñas, temporadas vacacionales o inicio de clases en el sistema educativo.

Dentro de la numérica también se encuentra lo que tiene que ver con la forma de contratación, pues como ya se ha mencionado la externalización forma parte de la flexibilidad interna, sin embargo cuando aquella versa sobre fuerza de trabajo, puede llegar a ser perjudicial a los derechos laborales de los trabajadores, aunque no por ello deja de catalogarse como flexibilidad, incluso en los casos de insourcing, en donde la producción se interioriza.

Si se analizan los tipos de flexibilidad mencionados con antelación, es posible percatarse que la flexibilidad laboral, además de siempre haber existido, si se respetan las normas de trabajo, no implica en modo alguno la violación de los derechos laborales en perjucio del trabajador.

Las distintas modalidades de flexibilidad mencionadas, desde luego pueden presentarse conjuntamente, de acuerdo a las necesidades y naturaleza del trabajo, por lo que las posibilidades son muchas, e inciden en el volúmen de empleo a nivel de un país o de una región en el mundo.



pp. $61-83$ 
ENFOQUES JURIDICOS

REVISTA MULTIDISCIPLINAR DEL CEDECS

ISSN 26832070

Número 04

julio-diciembre 2021
Artículo: "La reforma en materia de teletrabajo en México"

Jorge Martínez Martínez

DOI: https://doi.org/10.25009/ej.v0i04.2571

Por otro lado no debe confundirse la flexibilidad que se ha mencionado con la flexibilización de los derechos laborales, ya que flexibilizar éstos implica desde luego su incumplimiento, es decir la violación a las normas de trabajo.

Por fortuna, hasta la fecha, han permanecido muchos de los límites en la ley de la materia que han mantenido la pervivencia del derecho del trabajo, pues al flexibilizar en demasía, se inclina la balanza en favor de alguno de los factores de la producción:

La flexibilidad llevada a su último extremo, da paso a la desregulación, es decir, a la aplicación absoluta de la autonomía de la voluntad, - tanto por parte del patrón, como del trabajador-, para establecer las condiciones de trabajo, lo que en realidad sólo permite esta facultad (propio en cierto grado del derecho privado) al dueño de la empresa. Debe señalarse que esta situación no se ha llevado a cabo hasta la fecha en nuestro país de una manera formal y general, lo que no significa que en algunas áreas de la producción como el trabajo domicilio, el trabajo doméstico y otros que se realizan en algunos talleres y otras fuentes de empleo (y de sobre explotación y desfalco de la fuerza de trabajo) no sean una práctica común. (Lóyzaga, 1997: 47)

Las violaciones a los derechos de los trabajadores es un problema que se presenta constantemente, en virtud de que la realidad se impone, de manera que las normas de trabajo regulan de cierta manera algunos aspectos que no se aplican efectivamente, ya que la justicia no es automática, si no que se requiere que se haga valer; la aclaración resulta pertinente, ya que se ha expresado que la flexibilidad laboral ha sido positiva a través del tiempo, y que ha representado enormes ventajas para trabajadores y patrones, en beneficio desde luego de las fuentes de trabajo y de la productividad; no obstante la flexibilización de los derechos laborales, aunque puede traducirse en beneficio para el sector patronal, no debe permitirse o tolerarse, e incluso en el caso de algunos derechos, ha sido motivo de regulación en el TMEC.

\section{El trabajo a domicilio}

Desde que el trabajo comenzó a regularse en México y particularmente a partir de la federalización de la materia en 1931, se ha presentado una evolución que pretende incluir a diversas categorías de trabajo, lo que se evidencia desde la creación del apartado " $B$ " del artículo 123 constitucional (5 de diciembre de 1960) ya que además en su momento se intentó la creación de un apartado "C" en el año de 1976, (Martínez, 2006) sin que el intento tuviera éxito; no obstante lo mencionado pone de manifiesto la intención de una normativa diferenciada en materia de trabajo, si se toman como punto de partida las normas de trabajo constitucionales.

En la doctrina se ha discutido sobre el trabajo a domicilio, si en realidad se trata de trabajo conforme lo regulan las normas de la materia, si se presenta el elemento de la subordinación, así como los componentes de esta, que son el poder de mando y el deber de obediencia.



pp. $61-83$ 
ENFOQUES JURIDICOS

REVISTA MULTIDISCIPLINAR DEL CEDECS

ISSN 26832070

Número 04

julio-diciembre 2021
Artículo: “La reforma en materia de teletrabajo en México"

Jorge Martínez Martínez

DOI: https://doi.org/10.25009/ej.v0i04.2571

La definición actual de trabajo a domicilio se encuentra en el artículo 311 de la Ley Federal del Trabajo, donde se destaca en el diverso 312, que si la materia prima u objetos son vendidos por el patrón para ser confeccionados o transformados por el trabajador y posteriormente son vendidos al mismo patrón, constituye trabajo a domicilio.

Las labores se realizan en el domicilio del trabajador o en el lugar convenido, lo que a través del tiempo ha significado grandes ventajas para los patrones, sobre todo si el tipo de actividad lo permite, ya que de acuerdo a la especialización y a la naturaleza de las funciones del trabajador, no es aplicable en todos los casos; en México se ha puesto como ejemplo la elaboración de artesanías, los atuendos regionales o típicos, que permiten al patrón la venta de los productos, aunque en realidad no se paga por producto, sino que se paga un salario.

Este tipo de trabajo ha precarizado en muchas ocasiones las condiciones laborales de quienes lo llevan a cabo y ha sido objeto de estudio desde hace varias décadas, pues como ha sostenido Carlos Reynoso,

[...]otras investigaciones sobre el TAD en entidades federativas como Michoacán y Jalisco, precisan que las industrias involucradas realizan labores como la elaboración de prendas de tejido de punto, fabricación de diferentes tipos de calzado (y labores como la elaboración de tacones) artículos de cuero (balones, chamarras, cinturones) arreglos florales para novias [...] (Reynoso, 1992: 114).

En México el antecedente mas remoto sobre la regulación del trabajo a domicilio es del año de 1926, en la Ley del Trabajo del Estado de Oaxaca, normativa previa al capítulo XVIII de la Ley Federal del Trabajo de 1931; en ésta se señaló que a los trabajadores a domicilio les serían entregados artículos de fabricación y materias primas; en cambio en la ley de la materia de 1970, el trabajador a domicilio sería la persona que trabaja personalmente, con ayuda de miembros de su familia para un patrón. Posteriormente en fecha 10 de marzo de 1988, se incorpora como una de las categorías de trabajos especiales (Reynoso, 1992: 119) y finalmente en la regulación actual, el artículo 314 de la ley de la materia establece que los patrones pueden o no suministrar los útiles o materiales de trabajo.

Es curioso este tipo de trabajo, porque llega a situarse en el campo de la externalización de la producción, en donde los trabajadores pueden llevarse algún producto o material, para que, siguiendo las indicaciones, realicen la parte que les corresponda, se pueden suministrar o no los materiales, y por lo general no se les proporciona herramienta de trabajo, que se traduce en un ahorro para los patrones, pues el desgaste de los instrumentos lo absorben los trabajadores.

Se ha afirmado también la precarización del trabajo que puede producirse, pues se llega a convertir en una especie de esclavitud a domicilio, en donde pueden intervenir no sólo el trabajador, sino otros miembros de su familia, e incluso niños y tampoco se sujeta a horarios debido a su propia naturaleza.

Esta modalidad de trabajo ha existido desde hace mucho tiempo, y al igual que otras cuestiones del derecho laboral, su regulación ha enfrentado dificultades tanto para el

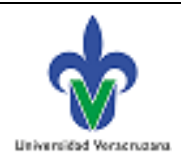

pp. $61-83$

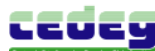


ENFOQUES JURIDICOS

REVISTA MULTIDISCIPLINAR DEL CEDECS

ISSN 26832070

Número 04

julio-diciembre 2021
Artículo: "La reforma en materia de teletrabajo en México"

Jorge Martínez Martínez

DOI: https://doi.org/10.25009/ej.v0i04.2571

reconocimiento de la relación de trabajo, así como para la obtención de datos sobre el particular:

Al respecto Octavio Lóyzaga y Verónica Curiel expresan:

Lo expuesto no es un problema nuevo, ya que el trabajo a domicilio, como se mencionó, es un fenómeno que se encuentra presente en la realidad de la sociedad desde hace más de 150 años; aunque ni en México ni en otros países hay cifras exactas, respecto a su año de origen, situación que se complica al encontrar lagunas legales que hacen más difícil su análisis e investigación. (Lóyzaga \& Curiel, 2014: 662)

El trabajo a domicilio y el teletrabajo, pueden tener en común, que se llevan acabo en el domicilio del trabajador, aunque la diferencia desde luego radica en el uso de las tecnologías de la información y comunicación, por lo que la regulación del teletrabajo sin duda era una tarea pendiente por regular en la ley de la materia.

El trabajo a domicilio lo define también el convenio número 177 de la Organización Internacional del Trabajo, de la manera siguiente:

Artículo 1.

A los efectos del presente convenio

(a) la expresión trabajo a domicilio significa el trabajo que una persona, designada como trabajador a domicilio, realiza:

(i) en su domicilio o en otros locales que escoja, distintos de los locales de trabajo del empleador;

(ii) a cambio de una remuneración;

(iii) con el fin de elaborar un producto o prestar un servicio conforme a las especificaciones del empleador, independientemente de quién proporcione el equipo, los materiales u otros elementos utilizados para ello, a menos que esa persona tenga el grado de autonomía y de independencia económica necesario para ser considerada como trabajador independiente en virtud de la legislación nacional o de decisiones judiciales;

(b) una persona que tenga la condición de asalariado no se considerará trabajador a domicilio a los efectos del presente Convenio por el mero hecho de realizar ocasionalmente su trabajo como asalariado en su domicilio, en vez de realizarlo en su lugar de trabajo habitual;

(c) la palabra empleador significa una persona física o jurídica que, de modo directo o por conducto de un intermediario, esté o no prevista esta figura en la legislación nacional, da trabajo a domicilio por cuenta de su empresa. (OIT, 2021)

Destaca en el inciso b) que no se considere como trabajador a domicilio, el asalariado que realiza ocasionalmente su trabajo de esta manera, en cuyo caso habría que determinar la circunstancia de "ocasional", ya que a partir de la pandemia de COVID-19, se han transformado las relaciones de trabajo; por ende, a más de un año, resulta discutible el punto, y para tener mayor certeza jurídica, las normas de trabajo deben definir lo que debe considerarse como "ocasional".

Finalmente se debe agregar que a pesar de los esfuerzos de la Organización Internacional del Trabajo, el Estado mexicano no ha ratificado hasta el momento el convenio número 177 (OIT, 2021) e igualmente faltan por ratificar otros, lo que en pleno paradigma constitucional de los derechos humanos, evidencia el camino que falta por recorrer para la regulación

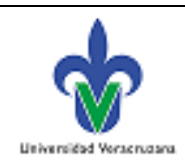

pp. $61-83$ 
ENFOQUES JURIDICOS

REVISTA MULTIDISCIPLINAR DEL CEDEGS

ISSN 26832070

Número 04

julio-diciembre 2021
Artículo: "La reforma en materia de teletrabajo en México"

Jorge Martínez Martínez

DOI: https://doi.org/10.25009/ej.v0i04.2571

adecuada y la tutela de los derechos de los trabajadores, así como también muestra la carencia de compromiso que México tiene, pues lo mismo sucede con la recomendación 184.

La reforma en materia de teletrabajo es un avance significativo; sin embargo, al mismo tiempo pueden realizarse acciones paralelas como es la ratificación del citado Convenio 177, aunque en la práctica podría invocarse, acorde a la interpretación que ha hecho la Corte Interamericana de Derechos Humanos, en sus diversas resoluciones, pues el Pacto de San José impone la obligación de respetar los derechos; aunado a que este tipo de argumentación es congruente con la evolución del Estado constitucional en México, y con los principios pro persona, así como el in dubio pro operario.

Por último en lo que concierne a este apartado, hay que considerar que debido a las circunstancias en las que se lleva acabo el trabajo a domicilio, la realidad ha prevalecido, y es difícil en muchos casos que siquiera se tenga conocimiento de la existencia de este tipo de trabajo, lo que complica el respeto a los derechos de los telerabajadores; por ello, se propone no considerarlo como trabajo especial, pues si bien por una parte es entendible que se lleva acabo de manera diferente, el tratamiento diferenciado no encuentra fundamento ni constitucional, ni axiológico.

\section{El teletrabajo en el contexto de la pandemia de COVID-19}

La pandemia de COVID-19 ha cambiado de facto las relaciones de trabajo, al igual que las formas de contratación, pues ha exigido una adaptación a las circunstancias, adversas en muchos casos, todo ello debido a cuestiones de salud.

Esta pandemia ha constituido un hito histórico para las relaciones de trabajo, al igual que lo han hecho las revoluciones industriales y los modelos de producción, pues a partir de ahora el mundo entero se ha tenido que adaptar al contexto que se ha presentado y en la medida de sus posibilidades, la población ha incursionado en el teletrabajo.

La Organización Internacional del Trabajo ha señalado en su Guía Práctica denominada El teletrabajo durante la pandemia de COVID-19 y después de ella, que como resultado de las medidas de confinamiento se produjeron aumentos significativos del teletrabajo:

Como resultado de las directivas de confinamiento decretadas por los gobiernos, casi 4 de cada 10 empleados en Europa comenzaron a teletrabajar (Eurofound, 2020). El aumento más significativo del teletrabajo tuvo lugar en los países más afectados por el virus y en los que el teletrabajo estaba bien desarrollado antes de la pandemia. En Finlandia, cerca del 60 por ciento de los empleados pasaron a trabajar desde casa. En Luxemburgo, los Países Bajos, Bélgica y Dinamarca, más del 50 por ciento, en Irlanda, Austria, Italia y Suecia, alrededor del 40 por ciento de los empleados teletrabajaban. En esos países, menos trabajadores vieron una reducción en su tiempo de trabajo. En promedio, en Europa, el 24 por ciento de los empleados que nunca habían trabajado desde casa comenzó a teletrabajar, en comparación con el 56 por ciento de los empleados que lo habían hecho ocasionalmente. No obstante, este salto en las cifras muestra que, con la tecnología, las herramientas (por ejemplo, de comunicación)



pp. $61-83$ 
ENFOQUES JURIDICOS

REVISTA MULTIDISCIPLINAR DEL CEDECS

ISSN 26832070

Número 04

julio-diciembre 2021
Artículo: "La reforma en materia de teletrabajo en México"

Jorge Martínez Martínez

DOI: https://doi.org/10.25009/ej.v0i04.2571

y la reorganización del trabajo adecuados, muchos más trabajos pueden realizarse a distancia de lo que se suponía anteriormente (OIT, 2021).

Lo que indudablemente presenta variaciones dependiendo del país de qué se trate, pues se ha evidenciado que el teletrabajo enfrenta como problema fundamental el contar con las herramientas tecnológicas que permitan realizarlo adecuadamente, pues impacta en los costos de las empresas; con independencia de que la regulación debe de establecer muy claramente las limitantes que impidan generar un ambiente de trabajo poco propicio, que afecte a la salud de los trabajadores.

Por otro lado teletrabajo representa diversas ventajas, tal como lo ha expresado el preámbulo del Real Decreto Ley 28/2020 de España, publicado en el Boletín Oficial del Estado en fecha 22 de septiembre del año 2020:

Además, el trabajo a distancia es fundamental para favorecer el asentamiento y la fijación de población en el medio rural, tal y como se señala en las Directrices Generales de la Estrategia Nacional frente al Reto Demográfico, aprobadas por Consejo de Ministros el 29 de marzo de 2019. Esta norma ha de servir para hacer factible la oportunidad de revertir la despoblación, de acuerdo con las características de los territorios que sufren el declive demográfico, como zonas rurales y remotas, o pequeños municipios. (Boletín Oficial del Estado [BOE] 2021).

De la misma forma se aprecia como problema el estrés que genera el uso de las tecnologías, no obstante destaca la adaptación y congruencia de este tipo de normas de trabajo en países como el mencionado, que por lo menos cuentan con una política demográfica definida, igualmente hay que establecer que la reforma en materia de teletrabajo en España, da cumplimiento a la Directiva 2019/1158 de la Unión Europea:

[...] de 20 de junio de 2019, relativa a la conciliación de la vida familiar y la vida profesional de los progenitores y los cuidadores y por la que se deroga la Directiva UE 2010/18 del Consejo, en la cual se establece un auténtico derecho a la conciliación de la vida laboral y familiar a través del uso de las formas flexibles de trabajo, incluidas las fórmulas de trabajo a distancia. (BOE, 2021)

Lo que de alguna manera se refleja en la regulación que se ha hecho en México, pues se trata de conciliar la vida laboral con la personal, y desde luego se contribuye a lograr el trabajo decente, que sigue siendo un tema pendiente en el país.

Previo al surgimiento de la pandemia de COVID-19, el teletrabajo representó una proporción muy baja en México, ya que de acuerdo a la Encuesta Nacional de Ocupación y Empleo, llevada a cabo por el INEGI en el cuarto trimestre del año 2018, se tuvo que poco más de 752 mil personas realizaban un trabajo relacionado con las tecnologías de la información y comunicación, en donde los hombres representaron el $82.9 \%$ y las mujeres el $17.1 \%$, que en su conjunto significaron el $1.4 \%$ de la población ocupada del país (Instituto Nacional de Estadística y Geografía [INEGI] 2021).

Con ello se entiende, más no justifica la demora en la reforma laboral en materia de teletrabajo, pues probablemente para los poderes del Estado era poco relevante el sector, a pesar de la dependencia tecnológica que indudablemente ha permeado cada vez más en



pp. $61-83$

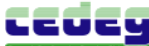


ENFOQUES JURIDICOS

REVISTA MULTIDISCIPLINAR DEL CEDECS

ISSN 26832070

Número 04

julio-diciembre 2021
Artículo: "La reforma en materia de teletrabajo en México"

Jorge Martínez Martínez

DOI: https://doi.org/10.25009/ej.v0i04.2571

la sociedad. Igualmente destaca la reducida participación que tuvieron las mujeres en este tipo de actividades relacionadas con la tecnología.

Del mismo modo y acuerdo a la Encuesta Telefónica sobre COVID-19 y Mercado Laboral (ECOVID-ML) llevada a cabo por el INEGI, con corte de información hasta el mes de abril del año 2020, se tiene que de 68.2 millones de habitantes que constituyen la población usuaria de teléfono, sólo 35 millones (51.3\%) integran la Población Económicamente Activa, y 33.2 millones (48.7\%) corresponden a la Población no Económicamente Activa. (INEGI ECOVID_ML, 2021)

La encuesta desde luego, como ya se ha apuntado, se basa en la población usuaria de teléfono, por lo que de inicio las cifras pudieran variar; aunque para efectos del tema que nos ocupa hay que tomar en cuenta que, si la población de que se trata ni siquiera es usuaria de teléfono, sus competencias laborales tecnológicas podrían ser reducidas.

De la citada Población Económicamente Activa, la población desocupada abierta, alcanzó la cifra de 2.1 millones de personas, que representa el 5.9\% de la PEA (INEGI ECOVID_ML, 2021) lo que indudablemente es problema a considerar en términos de desempleo.

Por otra parte, el $78.2 \%$ no se ausentó de su trabajo ni sufrió suspensión laboral alguna, mientras el $21.8 \%$ si se ausentó o su trabajo fue suspendido temporalmente, y de dicha cifra, el 92.9\% manifestó que la razón de ello fue el COVID-19 (INEGI ECOVID_ML, 2021).

\begin{tabular}{|c|c|c|c|c|}
\hline \multicolumn{5}{|c|}{ De 32.9 millones de personas ocupadas } \\
\hline $\begin{array}{c}\text { Trabaja } \\
\text { desde casa }\end{array}$ & $\begin{array}{c}\text { Disminuyó } \\
\text { su jornada de } \\
\text { trabajo }\end{array}$ & $\begin{array}{c}\text { Disminuyó } \\
\text { su ingreso }\end{array}$ & $\begin{array}{c}\text { Recibió } \\
\text { apoyo del } \\
\text { gobierno }\end{array}$ & $\begin{array}{c}\text { No ha } \\
\text { recibido apoyo }\end{array}$ \\
\hline $23 \%$ & $42 \%$ & $46 \%$ & $5 \%$ & $90 \%$ \\
\hline \multicolumn{3}{|c|}{ De ese 23\% que trabaja desde casa (7.7 millones de personas) } \\
\hline Tiene el equipo & \multicolumn{2}{c|}{$\begin{array}{c}\text { Cuenta con condiciones } \\
\text { necesario }\end{array}$} & $\begin{array}{c}\text { Ha tomado cursos de } \\
\text { capacitación para el trabajo }\end{array}$ \\
\hline $70 \%$ & \multicolumn{2}{c|}{$84 \%$} & $27 \%$ \\
\hline
\end{tabular}

(Elaboración propia con datos del INEGI ECOVID_ML, 2021: 11-12)

Lo que permite apreciar objetivamente el impacto que en materia de trabajo ha traído consigo la pandemia de COVID-19, pues hay que partir de que, de la población de referencia, sólo 32.9 millones de mexicanos se encuentran laborando; con las evidentes consecuencias económicas, a pesar de que los datos oficiales comprenden temporalmente un período reducido si se le compara con lo que ha durado la emergencia sanitaria. En el mismo sentido si el 70\% de los 7.7 millones de personas, son quienes cuentan con el equipo necesario para trabajar desde casa, se traduce en 5.39 millones, y 2.31 millones de trabajadores que carecen de herramientas o instrumentos para hacerlo, sin que de la encuesta pueda desprenderse que se cuente con las condiciones propicias, ya que como se ha expresado de



pp. $61-83$

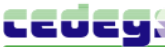


ENFOQUES JURIDICOS

REVISTA MULTIDISCIPLINAR DEL CEDECS

ISSN 26832070

Número 04

julio-diciembre 2021
Artículo: "La reforma en materia de teletrabajo en México"

Jorge Martínez Martínez

DOI: https://doi.org/10.25009/ej.v0i04.2571

acuerdo a la OIT, las jornadas se amplían, los trabajadores tienen a su cuidado menores, se carece de espacios adecuados o se presentan otro tipo de dificultades.

A las cifras citadas se agregan 5 millones de empleos perdidos temporalmente, de los cuales el $46 \%$ son de trabajadores sujetos a una relación de trabajo y el $53 \%$ a trabajadores independientes. (INEGI ECOVID_ML, 2021)

Asimismo se midió el impacto causado en las viviendas con población de 18 años y más usuaria de teléfono, hasta abril de 2020 , de lo cual se obtuvo que en el $30 \%$ de los casos, algún integrante de la vivienda perdió su trabajo y en el $65 \%$ de los hogares encuestados disminuyeron los ingresos. (INEGI ECOVID_ML, 2021)

Con la disminución de ingresos se produce desde luego un descenso en el nivel de vida que podían llevar las familias e impacta en el consumo de las mismas, lo que desacelera la recuperación de la economía.

Hay que tomar en cuenta las edades, la brecha tecnológica, la disposición que los trabajadores tienen para tele trabajar, pues a manera de ejemplo, al referirnos a la Universidad Veracruzana y en general al trabajo docente que se realiza de manera virtual en las instituciones de educación (sin ser propiamente teletrabajo) se encuentran dificultades de diversa naturaleza, como es la escasez de infraestructura tecnológica, tanto en los profesores, como por parte de los alumnos, sin menoscabo de considerar que no sólo es una cuestión de posibilidades económicas de las personas mencionadas, sino que se extiende a todo el país; a pesar de qué el derecho de acceso a Internet es considerado como derecho humano a partir de la reforma en materia de telecomunciaciones del año 2013.

\section{La reforma a la LFT en materia de teletrabajo}

En fecha 11 de enero del año 2021, se publicó en el Diario Oficial de la Federación, la reforma en materia de teletrabajo; la cual reformó el artículo 311; y se adicionó un capítulo XII Bis con los artículos 330-A; 330-B; 330-C; 330-D; 330-E; 330-F; 330-G; 330-H; 330-I; 330J y $330-K$ a la Ley Federal del Trabajo.

En lo que respecta al artículo 311, se suprimió el párrafo segundo y el tercero quedó como segundo.

La redacción anterior del artículo expresaba que sería considerado como trabajo a domicilio el que se realiza a distancia utilizando las tecnologías de la información y comunicación.

Luego entonces, la redacción anterior era muy escueta y prácticamente subsumió al teletrabajo en la categoría del trabajo a domicilio, lo cual es parcialmente cierto; no obstante la reforma del año 2012, resultó insuficiente, y las condiciones para su regulación actual fueron cambiando a partir del contexto actual, en donde indudablemente ha influido la pandemia de COVID-19.

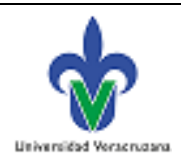

pp. $61-83$ 
ENFOQUES JURIDICOS

REVISTA MULTIDISCIPLINAR DEL CEDECS

ISSN 26832070

Número 04

julio-diciembre 2021
Artículo: “La reforma en materia de teletrabajo en México"

Jorge Martínez Martínez

DOI: https://doi.org/10.25009/ej.v0i04.2571

De acuerdo a María Aurora de la Concepción Lacavex Berumen "En realidad el teletrabajo no es un trabajo, sino una forma de trabajar, es decir, el contenido de la actividad que se teletrabajo puede ser cualquiera, desde las más sencillas y mecánicas, hasta lo más creativo." (Lacavex, 2009: 116)

En el capítulo XII bis de la ley de la materia, el artículo 330-A define al teletrabajo:

Artículo 330-A.- El teletrabajo es una forma de organización laboral subordinada que consiste en el desempeño de actividades remuneradas, en lugares distintos al establecimiento o establecimientos del patrón, por lo que no se requiere la presencia física de la persona trabajadora bajo la modalidad de teletrabajo, en el centro de trabajo, utilizando primordialmente las tecnologías de la información y comunicación, para el contacto y mando entre la persona trabajadora bajo la modalidad de teletrabajo y el patrón.

La persona trabajadora bajo la modalidad de teletrabajo será quien preste sus servicios personal, remunerado y subordinado en lugar distinto a las instalaciones de la empresa o fuente de trabajo del patrón y utilice las tecnologías de la información y la comunicación. (Diario Oficial de la Fedferación [DOF] 2021)

Como se aprecia, lo que caracteriza a este tipo de trabajo es la utilización de tecnologías de la información y comunicación de manera primordial, más no la única; y sigue teniendo en común con el trabajo a domicilio que se lleva a cabo en lugares distintos al establecimiento o establecimientos del patrón; se define igualmente al teletrabajador y finalmente, en el cuarto párrafo del mencionado artículo 330-A, se establece como limitante que para que resulten aplicables las disposiciones específicas aludidas, deben desarrollarse las relaciones de trabajo más del $40 \%$ en el domicilio de la persona trabajadora bajo esta modalidad, o en el domicilio que dicha persona elija.

Limitación fundamental, pues a contrario sensu, si las actividades se realizan hasta un $40 \%$ pero no más, no serán aplicables las disposiciones; incluso podría combinarse la realización de las labores en un lugar distinto al establecimiento o empresa o bien la utilización de las tecnologías, pero la ley exige que se presenten ambos elementos hasta el referido 40\%; entonces tendría que aplicarse la demás normativa, incluida la del trabajo a domicilio.

Asimismo, la ley de la materia establece que no será considerado teletrabajo, si se realiza de forma ocasional o esporádica; no obstante y como se ha comentado con anteación, la norma deja abierta la posibilidad de considerar lo que son estos calificativos; lo que sin duda puede relativizar su interpretación.

En el artículo 330-B se expresa que las condiciones de trabajo deben constar por escrito mediante un contrato, en donde cada una de las partes conservará un ejemplar y debe de contener además de lo establecido por el artículo 25 de la ley, el nombre, nacionalidad, edad, sexo, domicilio de las partes, naturaleza y características del trabajo, monto del salario, fecha, lugar y forma de pago, el equipo e insumos, en donde se debe incluir lo relacionado con las obligaciones de seguridad y salud para los teletrabajadores; la descripción y monto que se pagará a la persona bajo la modalidad de teletrabajo por

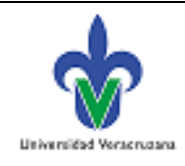

pp. $61-83$

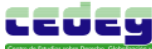


ENFOQUES JURIDICOS

REVISTA MULTIDISCIPLINAR DEL CEDECS

ISSN 26832070

Número 04

julio-diciembre 2021
Artículo: "La reforma en materia de teletrabajo en México"

Jorge Martínez Martínez

DOI: https://doi.org/10.25009/ej.v0i04.2571

concepto de pago, los mecanismos de pago y supervisión, duración y distribución de horarios, sin exceder los límites legales, así como las demás estipulaciones que establezcan las partes.

En el numeral 330-C se dispone que la modalidad de teletrabajo debe formar parte del contrato colectivo de trabajo, entregarse un ejemplar gratuito de éste a cada uno de los trabajadores y facilitarse los mecanismos de comunicación y difusión a los teletrabajadores para que conozcan los procedimientos de libertad sindical y negociación colectiva; por su parte el diverso 330-D establece que los patrones que no cuenten con contrato colectivo de trabajo, deberán regular el teletrabajo en sus reglamentos interiores de trabajo.

En el artículo 330-E se señalan las obligaciones especiales de los patrones, en las que se encuentran el proporcionar y mantener los equipos necesarios como equipos de cómputo, sillas, impresoras, el pago de los servicios de telecomunicación y la parte proporcional de la electricidad, llevar un registro de insumos en materia de seguridad y salud en el trabajo, implementar mecanismos para la seguridad de la información y datos; respetar el derecho a la desconexión de los teletrabajadores, cumplir con las obligaciones patronales en cuanto a la inscripción de sus trabajadores al régimen obligatorio del Seguro Social, capacitar a sus trabajadores, recibir los trabajos y pagar oportunamente los salarios.

En el diverso 330-F se enlistan las obligaciones de los teletrabajadores; que son el cuidar y conservar los materiales de trabajo, informar sobre los costos pactados sobre el uso de servicios de telecomunicaciones y consumo de electricidad, apegarse a las disposiciones de salud y seguridad en el trabajo, atender los mecanismos y sistemas operativos para la supervisión de sus actividades y atender políticas y mecanismos para proteger la información y los datos que manejen.

En el numeral $330-\mathrm{G}$ se establece que el cambio de la modalidad presencial al teletrabajo debe ser voluntario y por escrito, excepto en los casos de fuerza mayor debidamente acreditada; igualmente se señala el derecho a la reversibilidad estableciéndose la posibilidad de pactar los mecanismos y procesos para hacer válida esta manifestación de voluntad.

En relación al mencionado artículo debe enfatizarse la consideración de lo que conforme a la ley es considerado teletrabajo de manera esporádica, pues hay trabajo que hoy en día se subsume en esta categoría de manera material, sin que formalmente se haya establecido en las normas de trabajo que correspondan.

En el artículo 330-H se señala la obligación patronal de promover el equilibrio de la relación laboral para cumplir con lo señalado por la OIT como trabajo decente, pues debe haber igualdad de trato, seguridad social, acceso a mejores oportunidades laborales, al igual que los trabajadores presenciales, observarse la perspectiva de género y conciliar la vida personal de los teletrabajadores.

Ojalá y pueda materializarse lo previsto en el artículo en comento, pues el cumplimiento del trabajo decente sigue siendo una aspiración que se vislumbra lejana de lograr, pero que



pp. $61-83$

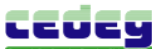


ENFOQUES JURIDICOS

REVISTA MULTIDISCIPLINAR DEL CEDECS

ISSN 26832070

Número 04

julio-diciembre 2021
Artículo: "La reforma en materia de teletrabajo en México"

Jorge Martínez Martínez

DOI: https://doi.org/10.25009/ej.v0i04.2571

sirve como guía para dirigir los esfuerzos hacia ello; desde luego que la equidad de género y la conciliación de la vida personal o familiar con el trabajo forma parte del trabajo decente, al guardar un equilibrio entre la vida privada y el trabajo, además, como ya se ha expresado esta disposición parece obedecer a una tendencia, o haber sido tomada de la legislación española, que tuvo lugar en septiembre del año 2020.

El artículo 330-I establece que los mecanismos, sistemas operativos, y tecnología para supervisar el trabajo deben ser proporcionales al objetivo y garantizar el derecho a la intimidad de las personas teletrabajadoras. En consecuencia sólo se colocarán cámaras y micrófonos de manera excepcional.

Parte de la problemática que se presenta es la invasión a la vida privada de los tele trabajadores, así como las dificultades para exigir el pago de horas extras; lo que deriva de la unión del domicilio del hogar con el del trabajo.

En el diverso 330-J, se refiere a las condiciones de seguridad y salud en el trabajo, que tendrán que establecerse en una Norma Oficial Mexicana, por parte de la Secretaría del Trabajo y Previsión Social, en la cual habrá que considerar distintos factores, como son los económicos y psicosociales, así como los que tengan que ver con el resguardo de la integridad física y mental de los tele trabajadores.

El último de los artículos relativos es el 330-K, en el que se contienen las atribuciones y deberes especiales de los inspectores de trabajo, qué son el comprobar el registro de insumos que los patrones proporcionan los teletrabajadores en cumplimiento a las obligaciones de seguridad y salud en el trabajo; la vigilancia de la igualdad salarial, y constatar el cumplimiento de las obligaciones especiales.

En el caso de este artículo, se espera funcione de manera adecuada, ya que muchas de las veces la inspección del trabajo en la realización de sus supervisiones, ha pasado por alto algunas cuestiones, en detrimento de los derechos laborales de los trabajadores.

La reforma es indudablemente un avance que puede considerarse sólo el punto de partida para la tutela de los derechos de los teletrabajadores, que por muchos años permanecieron sin regulación, y así asumieron las consecuencias de la omisión, pues se observa en ocasiones, que a pesar de la regulación estricta; la Ley Federal del Trabajo se ha seguido violando, y muestra de ello es el número creciente de asuntos, que se han planteado ante las autoridades laborales.

Después del análisis de la reforma, enseguida se enuncian algunos de los principales aspectos positivos y negativos.

Aspectos positivos:

- La disminución en algunos casos, de los costos para los empleadores, que propicia el crecimiento para las empresas y para la economía en general.

- La normatividad contribuye a lograr el trabajo decente.

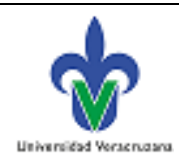

pp. $61-83$ 
ENFOQUES JURIDICOS

REVISTA MULTIDISCIPLINAR DEL CEDECS

ISSN 26832070

Número 04

julio-diciembre 2021
Artículo: "La reforma en materia de teletrabajo en México"

Jorge Martínez Martínez

DOI: https://doi.org/10.25009/ej.v0i04.2571

- Una mejor planeación en la demografía nacional, si es que se articula con la planeación en esta materia.

- Disminución de los tiempos de traslado, costos de transporte de los trabajadores (en algunos casos)

- Capacitación de los trabajadores en competencias tecnológicas.

- Adecuación de las normas de trabajo al contexto actual.

Aspectos negativos:

- El cumplimiento de la obligación de proporcionar a los tele trabajadores las herramientas tecnológicas, así como la capacitación, impactará en los costos de operación de algunas de las empresas.

- Complicaciones en el cumplimiento de la norma específicamente por cuanto hace a los descansos y el pago del tiempo extraordinario.

- Dificultades en materia procesal, en que respecta a la carga de la prueba del tiempo extraordinario, sobre todo en el caso de teletrabajo desconectado y sin supervisión con cámaras.

- Igualmente en materia procesal, las cargas probatorias en el caso de riesgos de trabajo.

- La violación al derecho a la intimidad y las consecuencias psicológicas como el estrés, aislamiento y pérdida de contacto físico de los tele trabajadores.

- La precarización de las condiciones de trabajo, al igual que ha ocurrido en el trabajo a domicilio.

Desde luego falta por avanzar, y antes de pensar en la necesidad de una regulación aun más pormenorizada, es necesario dar tiempo para que la reforma se materialice y de esta manera proponer las adecuaciones pertinentes, además de tomar en consideración que México se encuentra en la transición hacia el nuevo sistema de justicia laboral.

La pandemia de COVID-19 sirvió de catalizador para la reforma en materia de teletrabajo, pues como se expuso, en todo el mundo se optó por esta modalidad de acuerdo a las posibilidades de cada Estado; de acuerdo a las circunstancias, pues en Europa ya existía el Acuerdo Marco Europeo sobre Teletrabajo de 2002 (Boletín Oficial del Estado [BOE] 2020) con algunos aspectos mínimos que se han incorporado hoy en día en las reformas; con lo cual se pone de manifiesto que el derecho del trabajo debe ser dinámico y adaptarse a las transformaciones de los procesos de integración, globalización y regionalización, siempre en la búsqueda de la protección de las fuentes de trabajo y de los derechos de los factores de la producción.

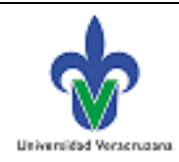

pp. $61-83$ 
ENFOQUES JURIDICOS

REVISTA MULTIDISCIPLINAR DEL CEDECS

ISSN 26832070

Número 04

julio-diciembre 2021
Artículo: "La reforma en materia de teletrabajo en México"

Jorge Martínez Martínez

DOI: https://doi.org/10.25009/ej.v0i04.2571

\section{Conclusiones}

1.- El trabajo a domicilio es una modalidad que ha existido desde hace muchos años, con una regulación que difícilmente ha respondido para la protección de los derechos de los trabajadores, quienes ante la necesidad de obtener el sustento para sus familias han laborado en condiciones muchas de las veces, desventajosas.

2.- El impacto de los modelos de producción, así como las revoluciones industriales ha sido definitivo para transformar los procesos productivos y las relaciones de trabajo, por lo que a partir de la cuarta revolución industrial (Industria 4.0), ha habido un cambio más profundo en cuanto a la utilización o incorporación de la tecnología en el trabajo.

3.- La pandemia de COVID-19 ha servido de catalizador para acelerar las reformas pendientes y adecuación de las normas de trabajo, que se habían postergado quizá porque las cifras permitían justificar qué se trataba de un aspecto menos relevante que otros, sin embargo la realidad se ha impuesto y de repente ante el confinamiento tuvieron que modificarse las relaciones de trabajo y adaptarse al contexto actual.

4.- La reforma de teletrabajo en México ha seguido las tendencias que se aprecian en Europa y otras partes del mundo, tal como lo ha señalado la Organización Internacional del Trabajo, con ello se ha materializado el reconocimiento de derechos y el establecimiento de obligaciones especiales acordes a esta modalidad de trabajo, que constituyen el punto de partida para el mejoramiento de las condiciones de trabajo de los teletrabajadores, ante un contexto complejo, y una realidad que indudablemente ha transformado los procesos productivos y las relaciones de trabajo.

5.- La aplicación de las normas de trabajo, al igual que cualquier reforma, representan un reto que hay que superar de manera gradual y paulatina, pues el artículo primero transitorio del Decreto por el cual se reforma el artículo 311 y se adiciona el capítulo XII bis de la Ley Federal del Trabajo en materia de teletrabajo, ha señalado que esta normativa entraría en vigor al día siguiente de su publicación, no obstante la justicia laboral en muchas de las ocasiones hay que hacerla valer en los tribunales ante su falta de observancia. Se espera que no sea el caso del teletrabajo, pues debido a lo reciente de la reforma, aun falta evaluar a que grado se cumplirá con las obligaciones señaladas en la misma.

6.- Finalmente se sostiene que la reforma en materia de teletrabajo dará buenos resultados a mediano y largo plazo, pues es evidente que obligó a los trabajadores a contar con competencias tecnológicas para realizar sus labores, lo cual los inserta y los capacita dentro de la denominada Industria 4.0, sin que deba perderse de vista que este tipo de transformaciones redundarán en una mejor sociedad y en la consecución del trabajo decente ya señalado por la Organización Internacional del Trabajo.

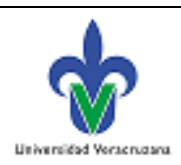

pp. $61-83$ 
ENFOQUES JURIDICOS

REVISTA MULTIDISCIPLINAR DEL CEDECS

ISSN 26832070

Número 04

julio-diciembre 2021
Artículo: "La reforma en materia de teletrabajo en México"

Jorge Martínez Martínez

DOI: https://doi.org/10.25009/ej.v0i04.2571

\section{Fuentes de consulta}

\section{Libros}

Lóyzaga De La Cueva, Octavio, 1997, La Flexibilización de los Derechos Laborales en la Recomposición del Capitalismo, México, UAM Azcapotzalco.

\section{Revistas}

Arancibia Fernández, F. 2011. "Flexibilidad laboral, elementos teórico conceptuales", Revista de Ciencias Sociales, no. 26.

Camacho Solís, J. I. 2021. "El teletrabajo, la utilidad digital por la pandemia del COVID-19", Revista Latinoamericana de Derecho Social, no 32., enero - junio.

Lacavex Berumen, M. A. C. 2009. “¿Es el teletrabajo, trabajo a domicilio? Una revisión hispanoamericana", Revista Latinoamericana de Derecho Social, no. 9., julio diciembre.

Lóyzaga De La Cueva, O., \& Curiel Sandoval, V. A. 2014. "El trabajo a domicilio en México", Alegatos - Revista Jurídica de La Universidad Autónoma Metropolitana, no. 88.

Piñero, F. J. 2004. "El modo de desarrollo industrial Fordista - Keynesiano: Características, crisis y reestructuración del capitalismo", Contribuciones a la Economía, junio.

Reynoso Castillo, C., 1992, "Trabajo a domicilio en México", Boletín Mexicano de Derecho Comparado, no. 73, enero - abril.

Taşkan, B., Karatop, B., Kubat, C. 2020. "Impacts of industrial revolutions on the enterprise performance managemet: A literature review", Journal of Business \& Management, vol. 26 Issue 1, marzo.

Tepe, G., \& Mucan Özcan, B. 2021. "Review and Bibliometric Analysis of Industry 4.0 in Social Sciences", Dumlupinar University Journal of Social, no. 67, en https://doi.org/10.512901/dpusbe.789178

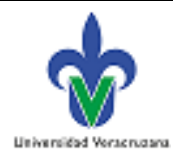

pp. 61-83 
ENFOQUES JURIDICOS

REVISTA MULTIDISCIPLINAR DEL CEDEGS

ISSN 26832070

Número 04

julio-diciembre 2021
Artículo: "La reforma en materia de teletrabajo en México"

Jorge Martínez Martínez

DOI: https://doi.org/10.25009/ej.v0i04.2571

Zuccarino, M. 2012. “Modelos estadounidense-fordista y japonés-toyotista: ¿Dos formas de organización productiva contrapuestas? Un estudio de caso: trabajadores bolivianos afiliados a la Federación Obrera Local (FOL) en la primera mitad del siglo XX", Historia Caribe, Volumen VII, Nº 21 - julio-diciembre.

\section{Legislación}

Boletín Oficial del Estado, 2020, en: https://www.boe.es/diario_boe/txt.php?id=BOE-A2020-11043

Decreto por el que se reforma el artículo 311 bis y se adiciona el capítulo XII Bis de la Ley Federal del Trabajo, Diario Oficial de la Federación del 11 de enero de 2021.

Real Decreto Ley 28/2020, en https://www.boe.es/diario_boe/txt.php?id=BOE-A-202011043

\section{Direcciones electrónicas}

http://dof.gob.mx/nota_detalle.php?codigo=5609683\&fecha=11/01/2021

https://www.ilo.org/dyn/normlex/es/f?p=NORMLEXPUB:11210:0::NO::P11210_COUNTRY _ID:102764

https://www.ilo.org/global/publications/lang--es/index.htm

https://www.ilo.org/wcmsp5/groups/public/---ed_protect/---protrav/--travail/documents/publication/wcms_758007.pdf

https://www.ilo.org/wcmsp5/groups/public/---ed_protect/---protrav/--travail/documents/publication/wcms_758007.pdf.

https://www.ilo.org/wcmsp5/groups/public/---ed_protect/---protrav/--travail/documents/publication/wcms_758007.pdf

https://www.inegi.org.mx/contenidos/investigacion/ecovidml/2020/doc/ecovid_ml_pres entacion.pdf)

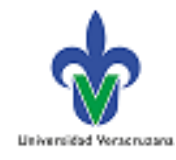

pp. $61-83$ 
ENFOQUES JURIDICOS

REVISTA MULTIDISCIPLINAR DEL CEDEGS

ISSN 26832070

Número 04

julio-diciembre 2021
Artículo: "La reforma en materia de teletrabajo en México"

Jorge Martínez Martínez

DOI: https://doi.org/10.25009/ej.v0i04.2571

https://www.inegi.org.mx/contenidos/saladeprensa/aproposito/2019/OcupaTIC2019_Nal .pdf

https://www.stunam.org.mx/8prensa/legadosindical/legado6/legado6-4.htm

Lozano, L. F., en

https://www.forbes.com.mx/negocios-ecommerce-se-acelera-1-de-cada-5-clientes-enamerica-latina-es-nuevo-a-partir-de-la-pandemia/, recuperado el 5 de agosto de 2020. 